\title{
Enhancement of Routing Protocols for Mobile Adhoc Network
}

\author{
Rajakumar.R ${ }^{\mathrm{a}, 1}$, PandianR ${ }^{\mathrm{b}}$, PremJacob .T $\mathrm{T}^{\mathrm{b}}$, Pravin .A $\mathrm{A}^{\mathrm{b}}$, Indumathi.P \\ ${ }^{a, 1}$ Assistant Professor, Dept of ECE, Sathyabama Institute of Science and Technology, \\ Chennai, India \\ ${ }^{b}$ Assistant Professor, Dept of ECE, Sathyabama Institute of Science and Technology, \\ Chennai, India \\ ${ }^{c}$ Professor, Dept of ECE, Madras institute of technology, Anna university, Chennai, \\ India
}

\begin{abstract}
The primaryaim of an ad-hoc network routing protocol is accurate and efficient route creation between node pairs so that messages may be delivered promptly. Route creation need to be done with reduced overhead and bandwidth. This paper presents a scheme to reduce bandwidth and power by the hibernation of nodes for a limited time.The effect of our proposal is then studied by simulation under various conditions and the analysis of the simulation results is done to comprehend the working of our protocol in various areas and how it fares in an application specific scenario.
\end{abstract}

Keywords. Mobile adhoc networks, energy, bandwidth, Network lifetime, fixed infrastructure, AODV

\section{Introduction}

MANET is a group of wireless mobile nodes forming a temporary network devoid of any central infrastructure.

\subsection{Characteristics of MANET}

Wireless, or one-hop networks, till very recently relied on a fixed structure, that is, network nodes linked to fixed infrastructure. Mobile ad-hoc networks (MANETs) provide many-hop communication, eventually allowing network nodes communicating via other nodes. In scenarios wherein networks are created and destroyed in an unplanned manner, MANET is the perfect choice. MANET has a changing topology. A mobile ad-hoc network is an assortment of devices with mobility having a transceiver, with an absent fixed infrastructure. The control and management of MANET is by the nodes.

\footnotetext{
${ }^{a, 1}$ Rajakumar R, Assistant Professor, Sathyabama Institute of Science and Technology, Chennai, India. E-mail: rrkmird@gmail.com
} 


\section{Enhancement of Routing Protocol}

The table-driven ad-hoc routing strategy is akin to the connectionless method where packets are forwarded, without consideration to how often the route(r)s are needed. It is based on an updating mechanism of routing table that takes into account the constant transmission of routing information. This is, untrue for on-demand routing protocols. When a node in on-demand protocol requires a route to a new sink, it has to wait till such route can be found out. The routing protocol that we have selected for enhancement is AODV[2]. The routing protocols available for adhoc networks have afew limitations in bandwidth, memory and memory [9-11]. AODV does not need much memory because it is a reactive protocol and hence does not store all the routing information in the table. Our protocol aims at improving the other two tradeoffs, there by all the limitations of adhoc routing protocols can be eliminated. Thus we chose AODV as our basic protocol on which we intend to implement the node hibernation algorithm to increase its efficiency[6].

\section{Hibernation of Nodes}

\subsection{Algorithm}

The implementation of hibernation of nodes to decrease the network traffic and to effectively equalize the power consumption by all the nodes is done as follows. Here, a node that has been recently in use goes to a hibernation state where it remains inactive for a specified amount of time. Thus the power usage is equalized among all the nodes. The following example will provide an idea of what we are trying to implement in the protocol. It depicts a scenario containing 21 nodes. There is no base station. It is adhoc in nature. Such a network can be constructed and broken down in very small time.

\subsection{Working}

Take node 1 as the originating node and node 21 as sink. The Path Discovery process is started when a source node is required to communicate with another node for which it has no routing data..Each node keeps two counters: a node sequence number and a broadcast id. The originating node (node 1) starts path discovery by broadcasting a route request (RREQ) packet to its neighbourhood. The RREQ comprises the below fields: $<$ source address, source sequence number, broadcast id, destination address, destination sequence number, hop count $>$

The Sink node (node 21) selects the shortest path based on the number of hops traversed and transmits a Route Reply (RREP) through the path chosen (unicast). On receipt of the RREP frame, the source node sends the Ready To Send (RTS) frame to the node. The sink node then transmits the Clear To Send (CTS) frame to the source through the chosen route. 


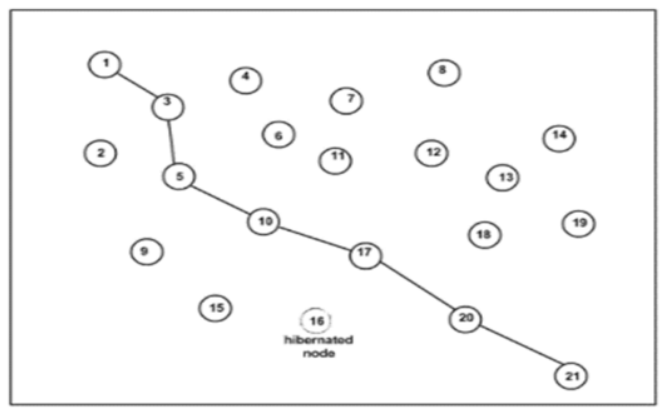

Figure 1. Hibernation of nodes

To conserve Power node 16 goes to hibernation mode, thus the route to node 21 is lost. The Source node again starts off the path discovery procedure and chooses the route with the shortest hop count. The new path selected has more hop counts than the previous one. If the node that goes to hibernation state was a node in critical path, then no route can be established to the destination node. A critical path is a path in which there is only one path connecting groups of nodes. In the above example, node 20 is in critical path, which is the only node connecting node 21 to the rest of the nodes.

\section{Simulation and Results}

Our protocol increases the network lifetime by effectively utilizing the battery power and it also results in a better spectral efficiency. The parameters that we have used for performance analysis are the packet delivery rate and the number of route requests generated. These parameters are indirectly indicative of the network lifetime and the bandwidth efficiency.

\subsection{Software used}

A network simulator as we are aware is a piece of software that mimics a network, without a network. Compared to the huge expense implied in setting up an entire test bed containing multiple networked computers, network simulators are far better.

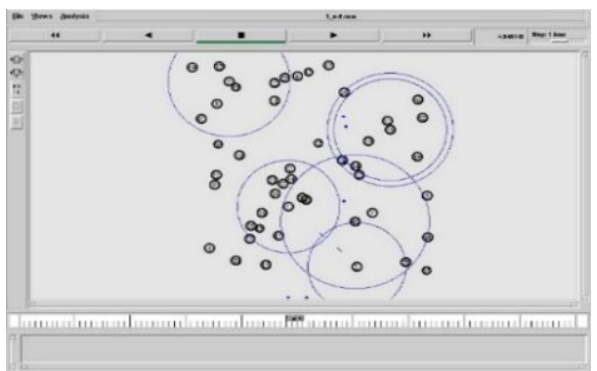

Figure 2.Nam screenshot of the scenario

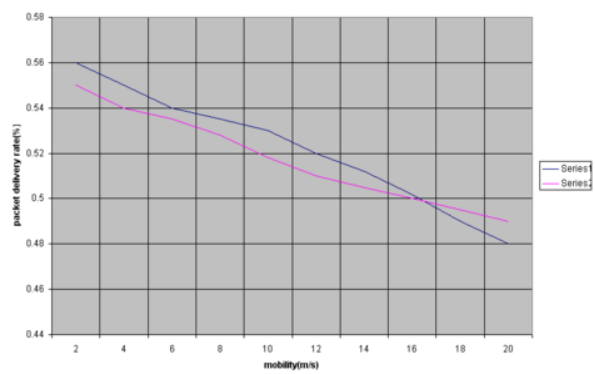

Figure 3.mobilityvs packet delivery rate 
Setdest is an independent utility available in NS2 to generate random motion for the nodes.Connections between TCP and CBR can be mobile nodes with a trafficscenario generator script. This script is in NS2 and is named ascbrgen.tcl. create CBR

\subsection{Mobility vs packet delivery rate}

From the simulation it was observed as in Fig.3, that the packet delivery rate goes down with the increase in the mobility from $(0-20) \mathrm{m} / \mathrm{s}$. the packet delivery rate is stated as follows Packet delivery rate $=$ number of packets received $/$ number of packets sent. It is seen from the simulation that not all the packets that are sent are successfully transmitted. A few of the packets are lost during the multiple hops. The packet delivery rate of the modified protocol is better than that of the basic AODV protocol. The packet delivery rate is higher for low to moderate mobility. If the mobility is increased further the packet delivery rate starts to decrease. This is mainly due to the increased mobile nature of the nodes. An increase of about 2 to 3 percentage is observed for mobility values of ( 0 to 15$) \mathrm{m} / \mathrm{s}$. The values of mobility can be varied by using a independent utility that is available inNS2 for creating random motion.

\subsection{Mobility vs number of route requests}

The number of route requests increases with the increase in the mobility. With increase in the mobility the topology becomes more chaotic thereby lots of route requests and packets get lost during transmission. The number of route requests generated is thus indicative of the control overhead. Thus by reducing the amount of number of route requests the bandwidth consumption is reduced and thereby more number of users can be accommodated within the same bandwidth. The observation shows that the number of route requests decreases by a fraction about 15 to 30 percentage of the original values produced by the basic protocol.

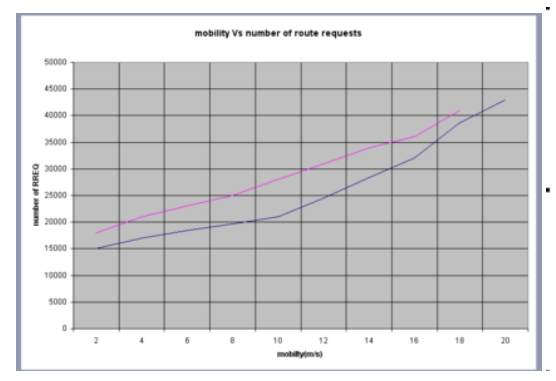

Figure 4.mobilityvs number of route requests

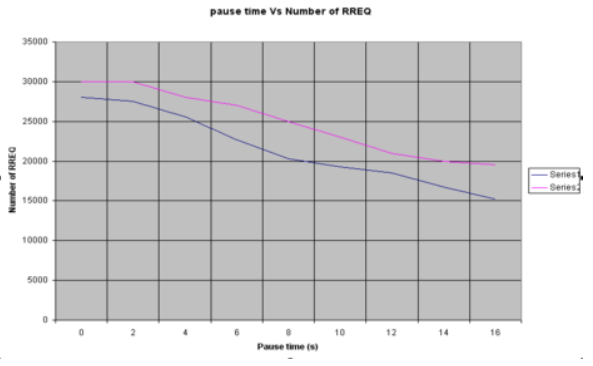

Figure 5.pause time vs number of route requests

\subsection{Pause time vs number of route request}

Pause time is defined as the infinitesimal small amount of time for which a node remains stationary in its position between two movements. This factor is also determining parameter in an adhoc network because increase in the pause time will make the network less dynamic and thereby the number of route request generated will be reduced. But for nodes moving in a same direction without changing direction the pause time will be less and thereby it will move away from the near by node. As a 
result the route request and other packets may get lost due to less pause time (Fig. 5). The value of the pause time used for simulation is varied from 0 to $20 \mathrm{~ms}$.

\section{Conclusion}

The observations of the above simulations concludes that our protocol fares better than the normal AODV protocol for low density networks and it starts to falter for high traffic networks and highly mobile networks. An increase of about 2 to $3 \%$ is observed in the packet delivery rate for low to moderate mobility values of ( 0 to 15$) \mathrm{m} / \mathrm{s}$.(Fig4) This value starts to decrease for higher mobility. The number of route requests lost decreases for the modified protocol when compared to the basic protocol and it varies from 6 to 24 percentage for varying pause times (Fig 5).From the above results it may be observed that the modified protocol performs better for networks where the node mobility is comparatively low. It also performs better with lower number of nodes. The performance starts to decrease for high traffic networks and high mobile networks. This results in application specific use for our protocol. MANET application includes deployment in battlefields to monitor battlefield survivability and for disaster recovery purposes. But for commercial application of adhoc networks like replacing the infrastructured services by adhoc services the modified protocol is not suitable for high traffic and scalability.. As a result the performance can be classified as working well under application specific scenarios. The modified protocol performs better in areas like battlefield survivability and disaster recovery[7], $\{8\}$. The future work includes expanding this idea for a hierarchical network like cluster head gateway switch routing and reducing the delay in hibernation by means of a route cache.

\section{Reference}

[1] Banerjee, Sumanand Misra, Archan (2002). Minimum Energy Paths for Reliable Communication in Multi-hop Wireless Networks, ACM MobiHOC.

[2] Cartigny, J., Simplot, D. and Stjmenovic, I, (2003). Localized minimum-energy broadcasting in ad-hoc networks, IEEEInfocom 2003.

[3] Christine Jones, E and et.al, (2001).A Survey of Energy Efficient Network Protocols for Wireless Networks, IETF Wireless Networks, Vol 7 , Issue 4.

[4] Elbatt, T. , Krishnamurthy, S. , Connors, D. and Dao, S. (2000). Power Management for Throughput Enhancement in Wireless Ad-Hoc Networks, ICC Magazine 2000.

[5] Elizabeth M.Royer and C-K Toh (1999).A review of current routing protocols for ad-hoc mobile wireless networks, IEEE Personal Communications Magazine.

[6] Kumar, P. (2003).Power Control and Clustering in Ad-Hoc Networks, IEEE Infocom, Vol 23, Issue 2.

[7] A. Jabbar, Mahamod Ismail, and Rosdiadee Nordin, (2014). On the Performance of the Current MANET Routing Protocols for VoIP, HTTP, and FTP Applications, Journal of Computer Networks and Communications, Feb, 2014.

[8] FahadTaha AL-Dhief, NaseerSabri, M.S. Salim , S. Fouad , S. A. Aljunid(2018).MANET Routing Protocols Evaluation: AODV, DSR and DSDV Perspective, MATEC Web of Conferences 150, 06024, pp 1-6.

[9] Ambeth Kumar V.D (2017). Efficient Routing for Low Rate Wireless Network a Novel Approach, International Journal of Image Mining, Vol. 2, Nos. 3/4, 2017, 2017.

[10] Nanagasabapathy.K et.al; .Validation system using smartphone luminescence.IEEE International Conference on Intelligent Computing, Instrumentation and Control Technologies (ICICICT), Pages: 235 - 239, 6-7 July 2017, Kannur, India.

[11] Ambeth Kumar .V.Det.al .Cloud enabled media streaming using Amazon Web Services.IEEE (ICSTM), Pages: 195-198, 2-4 Aug. 2017, Vel Tech University, Chennai, India (DOI: 10.1109/icstm.2017.8089150). 